Internat. J. Math. \& Math. Sci.

Vol. 23, No. 10 (2000) 723-728

S0161171200002623

(C) Hindawi Publishing Corp.

\title{
HOMOGENIZATION IN ELASTODYNAMICS WITH FORCE TERM DEPENDING ON TIME
}

\author{
PING WANG
}

(Received 28 September 1998)

\begin{abstract}
We extend the study on the homogenization problem for an elastic material containing a distributed array of gas bubbles to the case when the body force depends on time. By technically constructing an approximating sequence, we are able to show the convergence of semigroups and therefore prove the main result that such spongy material can be approximated by an elastic material without holes in the elastodynamics with the force term depending on time.
\end{abstract}

Keywords and phrases. Elastodynamics, homogenization, semigroup.

2000 Mathematics Subject Classification. Primary 35J50, 35B27; Secondary 76M50.

1. Introduction. Classical homogenization problems are considered in [2, 3]. Homogenization in elasticity and elastodynamics can be found in $[1,4,5,6]$. Especially in [5], we studied the homogenization problem for an elastic material containing a fine array of gas bubbles. The system considered was

$$
\rho u_{t t}^{\varepsilon}=\operatorname{div}\left(C \nabla u^{\varepsilon}\right)+f,
$$

where the displacement vector $u^{\varepsilon}$ is a function of $x$ and $t$ and the fourth-order elasticity tensor $C$, the density function $\rho$ and the body force $f$ are functions of $x$ and $x / \varepsilon$.

The homogenization problem in the case when $f$ depends on $t$ was not solved because the method used in [5] to derive the limiting system does not work.

Since in elastodynamics the case the body force depending on time $t$ is more interesting, we extend our study on homogenization problem of (1.1) to this more general and technically difficult case in this note. We assume that in (1.1) $C$ and $\rho$ are still functions of $x$ and $x / \varepsilon$ and the body force is a function of $x, x / \varepsilon, t$. To prove our main results, some results in [5] is applied, a sequence of function that approximates the body force is constructed and the convergence of semigroup operators is studied. We prove that $u^{\varepsilon}$, the solution of

$$
\rho\left(x, \frac{x}{\varepsilon}\right) u_{t t}^{\varepsilon}=\operatorname{div}\left(C\left(x, \frac{x}{\varepsilon}\right) \nabla u^{\varepsilon}\right)+f\left(t, x, \frac{x}{\varepsilon}\right),
$$

converges to $u^{0}$, the solution of

$$
\bar{\rho}(x) u_{t t}^{0}=\operatorname{div}\left(C^{0}(x) \nabla u^{0}\right)+\bar{f}(t, x)
$$

subject to appropriate boundary condition and initial conditions, where $C^{0}$ is the effective module and $\bar{\rho}$ and $\bar{f}$ are described later. 
This paper is arranged as follows: in Section 2, we define all notation and describe the problem. Since this is a work based on some results in [5], we list those results in this section. In Section 3, we construct of a sequence of function that approximates the body force and prove that convergence of semigroup operators. The main results of this paper are obtained by using the approximation technics and applying few results from [5].

2. Preliminaries. We denote the displacement vector by $u$. Assume $\Omega$ to be a bounded open set in $R^{3}$ with a Lipschitz continuous boundary. We define a periodic structure on $\Omega$ by tessellating $R^{3}$ with scaled and translated copies of the unit cell $Y=\Pi_{i=1}^{3}(0,1)$ as described below. Let an open subset $\mathcal{O}$ of $Y$ represent a hole in $Y$. We assume that the boundary of $O$ is $C^{1}$ and that the closure of $O$ does not intersect the boundary of $Y$. Denote $y=Y \backslash \mathcal{O}$. With each $x \in \Omega$ and $y \in Y$, we associate an elasticity tensor $C(x, y)$ satisfying the following hypotheses:

(1) $C(x, y)$ is a self-adjoint operator which maps second-order tensors to secondorder tensors for each $y$ in $Y$ and $x$ in $\Omega$. We assume the null space of $C$ consists of the skew-symmetric tensors, therefore for any second-order skew-symmetric tensor $W$, we have $C(x, y)[W]=0$.

(2) There exists a constant $\alpha>0$, independent of $x$ and $y$, such that

$$
E \cdot C(x, y)[E] \geq \alpha E \cdot E
$$

for all $y \in 9, x \in \Omega$ and every symmetric $E$. That is, $C(x, y)$ is uniformly positive definite in solid part in the space of second-order symmetric tensors.

(3) There exists a $\lambda>0$, such that, for $y \in \mathbb{0}$,

$$
C[E]=\lambda(\operatorname{tr} E) I,
$$

where $I$ is a second-order unit tensor.

(4) $C(x, y)$ is a bounded, measurable function of $y$ in $Y$ and a continuous function of $x$ in $\Omega$.

We consider the function $C(x, x / \varepsilon)$ in $\Omega$ as the elasticity tensor for a linearly elastic material. The elastic material encloses an array of gas-filled holes congruent to $\varepsilon \mathcal{O}$. Certainly $\partial \Omega$ may intersect some holes. Let $O_{\varepsilon}$ be formed by the union of all holes which are completely contained in $\Omega$. Denote $\Omega_{\varepsilon}=\Omega \backslash 0_{\varepsilon}$. In our problem, $\Omega_{\varepsilon}$ is the solid part while $O_{\varepsilon}$ is the gas part. We can easily derive similar assumptions on $C(x, x / \varepsilon)$ from assumptions (1)-(4). For simplicity, we just refer to assumptions (1)-(4) as assumptions on $C(x, x / \varepsilon)$ later on. We also introduce a product space $H=H_{0}^{1}(\Omega) \times L^{2}(\Omega)$ equipped with usual Sobolev norm $\|Q\|_{H}=\|u\|_{H_{0}^{1}(\Omega)}+\|v\|_{L^{2}(\Omega)}$ for $Q=(u, v)^{T} \in H$, where the superscript $T$ denotes the transpose. For $(g(x), h(x))^{T} \in H$, we consider the behavior, as $\varepsilon \rightarrow 0$, of the following system:

$$
\begin{aligned}
\rho\left(x, \frac{x}{\varepsilon}\right) u_{t t}^{\varepsilon} & =\operatorname{div}\left(C\left(x, \frac{x}{\varepsilon}\right) \nabla u^{\varepsilon}\right)+f\left(t, x, \frac{x}{\varepsilon}\right) \quad \text { in } \Omega \times R_{+} ; \\
u^{\varepsilon} & =0 \quad \text { on } \partial \Omega \times R_{+} ; \\
u^{\varepsilon}(0, x) & =g(x) ; \quad u_{t}^{\varepsilon}(0, x)=h(x) \quad \text { in } \Omega,
\end{aligned}
$$


where $\rho(x, x / \varepsilon)$ is the density function with the properties that it is integrable and that $\rho(x, x / \varepsilon)>k>0$ ( $k$ is a constant) for all $x$ in $\Omega$.

Let $v=u_{t}$ and $Q=(u, v)^{T}$. Then equations (2.3), (2.4), and (2.5) then could be rewritten as

$$
Q_{t}=\mathscr{C}_{\varepsilon} Q+F_{\varepsilon}(t) \quad \text { in } \Omega \times R_{+} ;\left.\quad Q\right|_{t=0}=(g, h)^{T} \quad \text { in } \Omega,
$$

where $F_{\varepsilon}(t)=(0, f(t, x, x / \varepsilon))^{T}, \mathscr{C}_{\varepsilon}$ is the following operator on $H$ :

$$
\mathscr{b}_{\varepsilon}=\left(\begin{array}{cc}
0 & 1 \\
\frac{1}{\rho(x, x / \varepsilon)} \operatorname{div}\left(c\left(x, \frac{x}{\varepsilon}\right) \nabla\right) & 0
\end{array}\right)
$$

with domain $D\left(\mathscr{C}_{\varepsilon}\right)=\left\{Q=(u, v)^{T} \in H\right.$ such that $\mathscr{C}_{\varepsilon} Q$ belongs to $\left.H\right\}$. With all these notation and assumptions, we are ready to list some useful results from [5].

LEMMA 2.1. Under assumptions (1)-(4) on $C(x, x / \varepsilon), \mathscr{b}_{\varepsilon}$ generates in $H$ a $C_{0}$ semigroup operator $S_{\varepsilon}(t)$ such that

$$
\left\|S_{\varepsilon}(t) Q\right\| \leq\|Q\|
$$

for any $Q \in H$. So, for any $G=(g, h)^{T} \in H, S_{\varepsilon} G \in H$ is a solution of system

$$
Q_{t}=\mathscr{C}_{\varepsilon} Q \quad \text { in } \Omega \times R_{+} ;\left.\quad Q\right|_{t=0}=G \quad \text { in } \Omega .
$$

LEMMA 2.2 (unit cell problem). Under assumptions (1)-(4) on $C(x, x / \varepsilon)$, for any constant second-order symmetric tensor $\Lambda$, there exists a weak solution, $w^{\Lambda}$, to the following differential system:

$$
\begin{gathered}
-\operatorname{div}\left(C(x, y)\left[\nabla_{y}\left(w^{\Lambda}+\Lambda y\right)\right]\right)=0, \quad y \in Y, \\
\int_{Y} w^{\Lambda} d y=0, \quad w^{\Lambda} \text { is a } Y \text {-periodic } H^{1} \text { function }
\end{gathered}
$$

with $x$ as a parameter in $\Omega$.

LEMMA 2.3. $S_{\varepsilon}(t) G \rightarrow S(t) G$ in $L_{\infty}\left(R_{+}, H\right)$ as $\varepsilon \rightarrow 0$, where $G=(g, h)^{T} \in H$ and $S(t)$ is a semigroup operator generated by

$$
\mathscr{C}_{0}=\left(\begin{array}{cc}
0 & 1 \\
\frac{1}{\bar{\rho}(x)} \operatorname{div}\left(C^{0}(x) \nabla\right) & 0
\end{array}\right)
$$

in which $\bar{\rho}=(1 /|Y|) \int_{Y} \rho(x, y) d y$ and $C^{0}$ is defined by the following

$$
C^{0}[\Lambda]=\frac{1}{|Y|} \int_{Y} C(x, y)\left(\nabla_{y}\left(w^{\Lambda}+\Lambda y\right)\right)
$$

for any constant second-order symmetric tensor $\Lambda$ and $w^{\Lambda}$ is obtained in Lemma 2.2.

LEMMA 2.4. If $f(x, x / \varepsilon) \in L^{2}(\Omega)$, then, as $\varepsilon \rightarrow 0$,

$$
\int_{0}^{t} S_{\varepsilon}(t-s) F^{\varepsilon}(x) d s \rightarrow \int_{0}^{t} S(t-s) \bar{F}(x) d s,
$$

where $F^{\varepsilon}(x)=(0, f(x, x / \varepsilon))^{T}, \bar{F}(x)=(0, \bar{f}(x))^{T}$ with $\bar{f}(x)=(1 /|Y|) \int_{Y} f(x, y) d y$. 
Proofs of Lemmas 2.1, 2.2, 2.3, and 2.4 can be found in [5]. One can easily show that the solution of (2.6) can be expressed as

$$
Q_{\varepsilon}(t, x)=\left(u^{\varepsilon}(t, x), v^{\varepsilon}(t, x)\right)^{T}=S_{\varepsilon} G(x)+\int_{0}^{t} S_{\varepsilon}(t-s) F\left(s, x, \frac{x}{\varepsilon}\right) d s .
$$

3. Convergence of semigroups. When the force depends on time variable, the method used in [5] to derive the limit system cannot be applied. The problem is to deal with the integral in (2.15). There we have a convolution term whose behavior is not clear if we directly send $\varepsilon$ to zero on both sides of (2.15) in order to obtain an equation which the limiting solution may satisfy. We should look for a new way to get around this difficulty. The idea is to choose an approximation sequence of the force function in such a way that functions in this sequence are piecewise constants with respect to time. Then we can apply Lemma 2.4 to each function in this sequence to study the limiting behaviour of all terms in (2.15).

Let us first prove the following technical lemma.

LEMMA 3.1. For $f(t, x, x / \varepsilon) \in C\left(0, T ; L^{2}(\Omega)\right)$, there exists a sequence $f_{n}(t, x, x / \varepsilon) \in$ $L^{1}\left(0, T ; L^{2}(\Omega)\right)$ such that $f_{n}(t, x, x / \varepsilon) \rightarrow f(t, x, x / \varepsilon)$ in $L^{1}\left(0, T, L^{2}(\Omega)\right)$ as $n \rightarrow \infty$. Moreover, $\left\{f_{n}\right\}$ are piecewise constant functions with respect to $t$.

Proof. Let us define, for integers $n$ and $j=1,2, \ldots, n$,

$$
f_{j}\left(t, x, \frac{x}{\varepsilon}\right)= \begin{cases}0 & {\left[0, \frac{j-1}{n} T\right)} \\ f\left(\frac{j}{n} T, x, \frac{x}{\varepsilon}\right) & {\left[\frac{j-1}{n} T, \frac{j}{n} T\right]} \\ 0 & \left(\frac{j}{n} T, T\right]\end{cases}
$$

and define $\chi_{j}$ as the following:

$$
\chi_{j}(t)= \begin{cases}1 & \text { on }\left(\frac{j-1}{n} T, \frac{j}{n} T\right) \\ 0 & \text { elsewhere. }\end{cases}
$$

Then take $f_{n}(t, x, x / \varepsilon)=\sum_{j=1}^{n} \chi_{j}(t) f_{j}(t, x, x / \varepsilon)$. It is obvious that the lemma follows.

Let us denote $F(t, x, x / \varepsilon)=(0, f(t, x, x / \varepsilon))^{T}\left(\in H_{0}^{1}(\Omega) \times C\left(0, T ; L^{2}(\Omega)\right)\right)$. We can show the following lemma.

LEMMA 3.2. Assume $f(t, x, x / \varepsilon) \in C\left(0, T ; L^{2}(\Omega)\right)$ and $f(t, x, y)$ is periodic in $y$ with period 1. We have the following convergence as $\varepsilon \rightarrow 0$ :

$$
\int_{0}^{t} S_{\varepsilon}(t-s) F\left(s, x, \frac{x}{\varepsilon}\right) d s \rightarrow \int_{0}^{t} S_{0}(t-s) \bar{F}(s, x) d s,
$$

where $\bar{F}(t, x)=(0, \bar{f}(t, x))^{T}$ with $\bar{f}(t, x)=(1 /|Y|) \int_{Y} f(t, x, y) d y$. 
Proof. Let us apply Lemma 3.1 to $F(t, x, x / \varepsilon)$. Then there exists a sequence of function $F_{n}(t, x, x / \varepsilon) \rightarrow F(t, x, x / \varepsilon)$ in $H_{0}^{1}(\Omega) \times L^{1}\left(0, T, L^{2}(\Omega)\right)$ and $F_{n}(t, x, x / \varepsilon)$ is a piecewise constant function with respect to variable $t$. It is a simple fact that, since $f(t, x, \cdot)$ is periodic,

$$
F\left(t, x, \frac{x}{\varepsilon}\right) \longrightarrow \bar{F}(t, x) \quad \text { in } H_{0}^{1}(\Omega) \times C\left(0, T, L^{2}(\Omega)\right),
$$

where $\bar{F}(t, x)=(0, \bar{f}(t, x))^{T}$ with $\bar{f}(t, x)=(1 /|Y|) \int_{Y} f(t, x, y) d y$. Now consider

$$
\begin{aligned}
\int_{0}^{t} S_{\varepsilon}( & -s) F\left(s, x, \frac{x}{\varepsilon}\right) d s-\int_{0}^{t} S_{0}(t-s) \bar{F}(s, x) d s \\
= & \int_{0}^{t}\left(S_{\varepsilon}(t-s) F\left(s, x, \frac{x}{\varepsilon}\right)-S_{\varepsilon}(t-s) F_{n}\left(s, x, \frac{x}{\varepsilon}\right)\right) d s \\
& +\int_{0}^{t}\left(S_{\varepsilon}(t-s) F_{n}\left(s, x, \frac{x}{\varepsilon}\right)-S_{0}(t-s) F_{n}\left(s, x, \frac{x}{\varepsilon}\right)\right) d s \\
& +\int_{0}^{t}\left(S_{0}(t-s) F_{n}\left(s, x, \frac{x}{\varepsilon}\right)-S_{0}(t-s) \bar{F}_{n}\left(s, x, \frac{x}{\varepsilon}\right)\right) d s \\
& +\int_{0}^{t}\left(S_{0}(t-s) \bar{F}_{n}\left(s, x, \frac{x}{\varepsilon}\right)-S_{0}(t-s) \bar{F}\left(s, x, \frac{x}{\varepsilon}\right)\right) d s .
\end{aligned}
$$

On the right-hand side of (3.4), applying Lemmas 2.1 and 3.1 to the first and the last integrals, Lemma 2.4 to the second integral and Lemma 2.1 and (3.4) to the third integral completes the proof of this lemma.

Applying Lemmas 2.3 and 3.2 to the right-hand side of (2.15), we conclude that, as $\varepsilon \rightarrow 0, Q_{\varepsilon}(t, x)$ converges weak star to $Q_{0}(t, x)$ in $L_{\infty}\left(R_{+}, H_{0}^{1}(\Omega)\right)$, where

$$
Q_{0}(t, x)=\left(u^{0}(t, x), v^{0}(t, x)\right)^{T}=S_{0}(t) G(x)+\int_{0}^{t} S_{0}(t-s) \bar{F}(s, x) d s .
$$

Therefore we prove the following theorem.

THEOREM 3.1. Under assumptions (1)-(4) of Section 2 on the elasticity coefficient $C(x, x / \varepsilon)$, for $g(x) \in H_{0}^{1}(\Omega), h(x) \in L^{2}(\Omega), f(t, x, x / \varepsilon) \in C\left(0, T ; L^{2}(\Omega)\right)$ as described in Lemma 3.2, the solution of (2.3), (2.4), and (2.5), $u^{\varepsilon}(t, x)$, converges weak star in $L_{\infty}\left(R_{+}, H_{0}^{1}(\Omega)\right)$ to $u^{0}$ defined by (3.6), the solution of

$$
\begin{aligned}
\bar{\rho}(x) u_{t t}^{0} & =\operatorname{div}\left(C^{0}(x)\left[\nabla u^{0}\right]\right)+\bar{f}(t, x) \quad \text { in } \Omega \times R_{+} ; \\
u^{0} & =0 \quad \text { on } \partial \Omega \times R_{+} ; \\
u^{0}(0, x) & =g(x) ; \quad u_{t}^{0}(0, x)=h(x) \quad \text { in } \Omega,
\end{aligned}
$$

where $\bar{\rho}(x)$ and $C^{0}$ are given in Section 2 and $\bar{f}(t, x)$ in Lemma 3.2.

\section{REFERENCES}

[1] A. Bensoussan, J. L. Lions, and G. Papanicolaou, Asymptotic Analysis for Periodic Structures, North-Holland Publishing Co., Amsterdam, 1978. MR 82h:35001. Zbl 404.35001.

[2] D. Cioranescu and J. S. J. Paulin, Homogenization in open sets with holes, J. Math. Anal. Appl. 71 (1979), no. 2, 590-607. MR 81j:35017. Zbl 427.35073. 
[3] J. L. Lions, Some Methods in the Mathematical Analysis of Systems and their Control, Kexue Chubanshe (Science Press), Beijing; Gordon \& Breach Science Publishers, New York, 1981, Beijing, 1981. MR 84m:49003. Zbl 542.93034.

[4] O. A. Oleĭnik, G. A. Iosifyan, and G. P. Panasenko, Asymptotic expansion of solutions of a system of elasticity theory in perforated domains, Mat. Sb. (N.S.) 120(162) (1983), no. 1, 22-41, 143. MR 84h:35022.

[5] P. Wang, Homogenization for inhomogeneous elastic body containing gas bubbles, Appl. Anal. 44 (1992), no. 1-2, 1-20. MR 95f:35026. Zbl 737.73010.

[6] _ The limiting case of zero shear modulus in linear elasticity, J. Elasticity 38 (1995), no. 2, 121-132. MR 97a:73025. Zbl 828.73020.

Wang: Department of Mathematics, Pennsylvania State University, Schuylkill HAVEN, SCHUYLKILL, PA 17972, USA

E-mail address: pxw10@psu.edu 


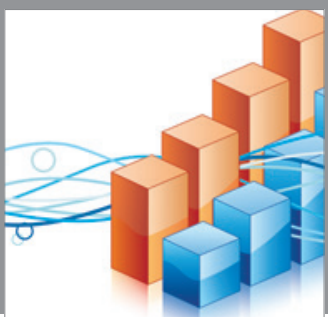

Advances in

Operations Research

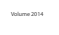

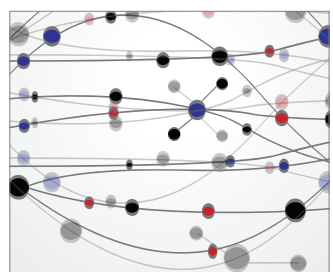

\section{The Scientific} World Journal
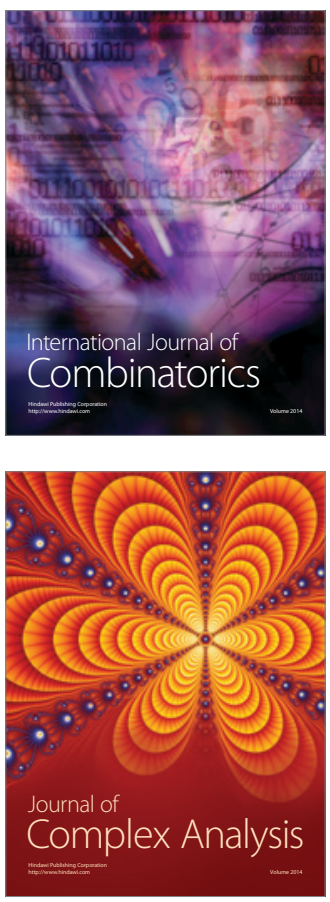

International Journal of

Mathematics and

Mathematical

Sciences
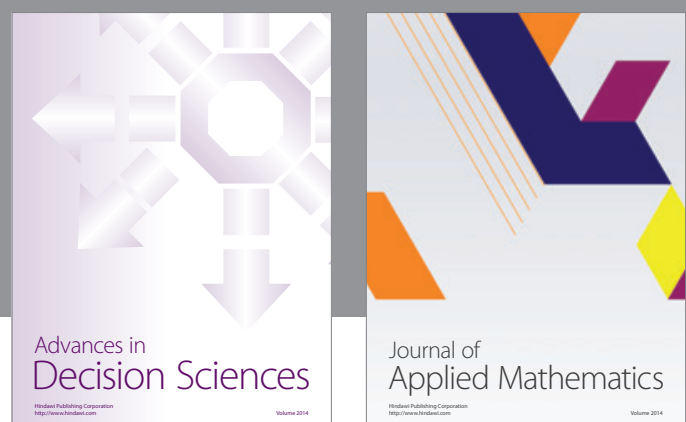

Journal of

Applied Mathematics
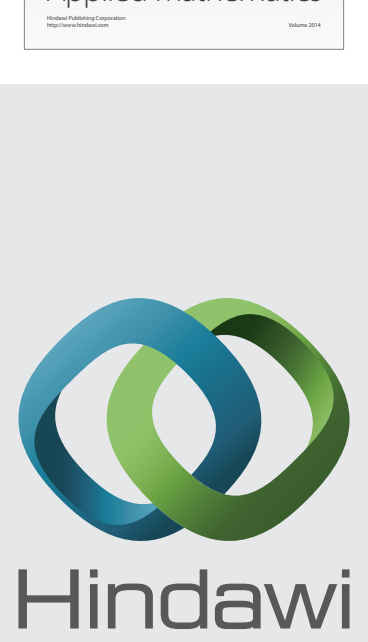

Submit your manuscripts at http://www.hindawi.com
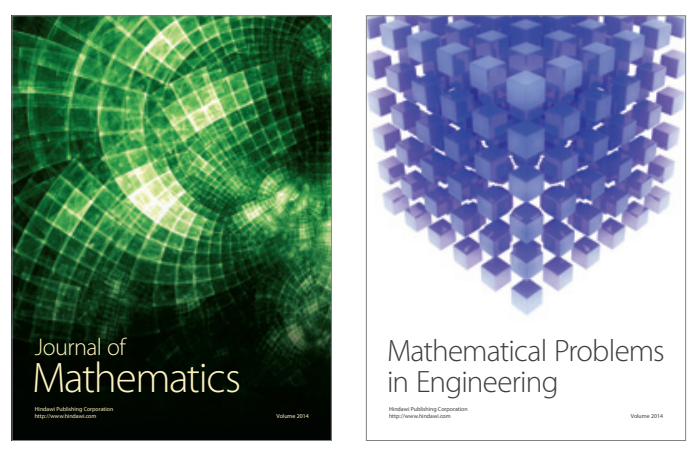

Mathematical Problems in Engineering
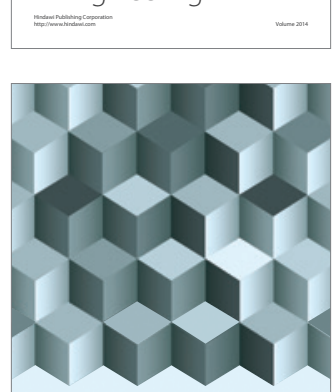

Journal of

Function Spaces
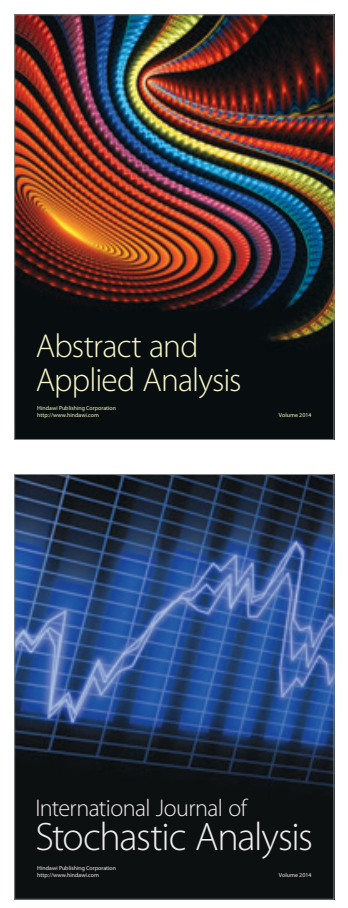

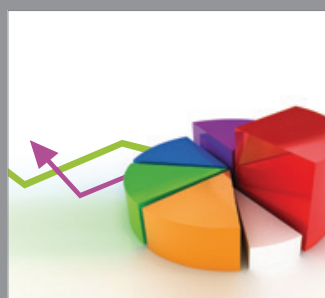

ournal of

Probability and Statistics

Promensencen
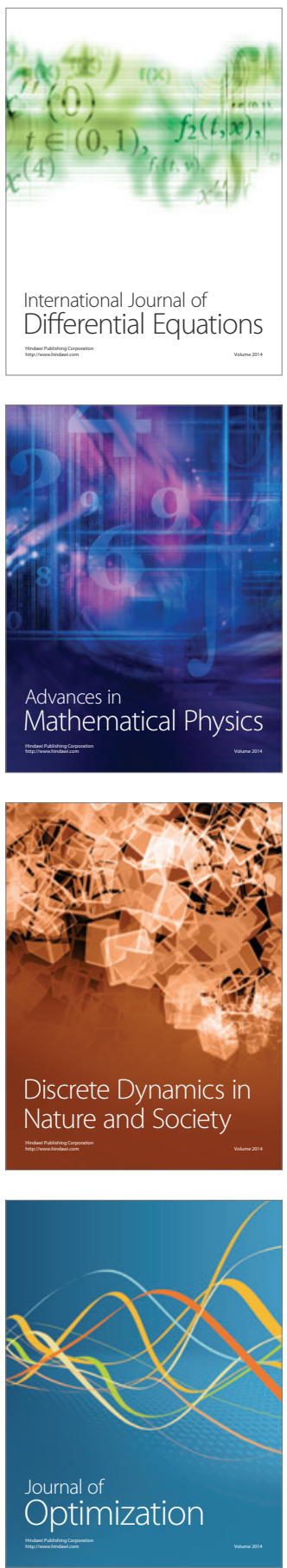